\title{
Anti-Thrombotic Effects of a Nitric Oxide-releasing, Gastric-sparing Aspirin Derivative
}

John L. Wallace, * Webb McKnight, * Piero Del Soldato, ” Anwar R. Baydoun, ${ }^{\mathbf{a}}$ and Giuseppe Cirino"

${ }^{*}$ Department of Pharmacology and Therapeutics, University of Calgary, Calgary, Alberta, Canada; ${ }^{\ddagger}$ NicOx Ltd., London, U.K.;

${ }^{8}$ Vascular Biology Research Centre, Biomedical Sciences Division, King's College, London, U.K.; and "Department of Experimental

Pharmacology, University of Naples, Naples, Italy

\begin{abstract}
Effects of a nitroxybutylester derivative of aspirin (NCX 4215 ) on platelet aggregation and prostanoid synthesis were compared to the effects of aspirin. NCX 4215 was approximately seven times more potent than aspirin as an inhibitor of thrombin-induced human platelet aggregation in vitro, but did not inhibit platelet thromboxane synthesis or gastric prostaglandin synthesis. NCX 4215 released nitric oxide when incubated in the presence of platelets and increased platelet levels of cGMP within 10 min of exposure, while aspirin did not. The anti-aggregatory effects of NCX 4215 in vitro were significantly attenuated by $10 \mu \mathrm{M}$ hemoglobin. In ex vivo studies of ADP- or collagen- or thrombin-induced rat platelet aggregation, aspirin and NCX 4215 had comparable inhibitory effects $3 \mathrm{~h}$ after administration. Aspirin (10-120 $\mathrm{mg} / \mathrm{kg}$ ) caused extensive hemorrhagic erosion formation in the stomach of the rat within $3 \mathrm{~h}$ of oral administration, while NCX 4215 did not produce significant damage at doses of up to $300 \mathrm{mg} / \mathrm{kg}$, nor when given daily for two weeks at $166 \mathrm{mg} / \mathrm{kg}$. NCX 4215 did not alter systemic arterial blood pressure when administered intravenously to the rat. These studies demonstrate that NCX 4215 has comparable or enhanced anti-thrombotic activity to that of aspirin, but does not cause gastric damage or alter systemic blood pressure. The anti-thrombotic actions of NCX 4215 are, at least in part, due to generation of nitric oxide. (J. Clin. Invest. 1995. 96:2711-2718.) Key words: thrombosis • ulcer - NSAID • platelet aggregation • thromboxane
\end{abstract}

\section{Introduction}

Aspirin is increasingly used for anti-thrombotic prophylaxis, particularly in patients who have had a myocardial infarction or angina pectoris, but also in healthy individuals in an attempt to prevent myocardial infarction (1). The beneficial effects of aspirin are attributable to its ability to irreversibly inhibit the enzyme cyclo-oxygenase, thereby preventing the formation of the pro-aggregatory, vasoconstrictor substance, thromboxane $A_{2}$

Address correspondence to John L. Wallace, Ph.D., Department of Pharmacology and Therapeutics, University of Calgary, 3330 Hospital Drive NW, Calgary, AB, T2N 4N1, Canada. Phone: 403-220-4539; FAX: 403270-3353; e-mail: wallacej@acs.ucalgary.ca

Received for publication 1 May 1995 and accepted in revised form 29 August 1995.

J. Clin. Invest.

(C) The American Society for Clinical Investigation, Inc.

$0021-9738 / 95 / 12 / 2711 / 08 \$ 2.00$

Volume 96, December 1995, 2711-2718
(2). Because the platelet is unable to generate new cyclo-oxygenase, the inhibitory effects of aspirin persist for the 8-10-d life span of the platelet. Activation of platelets and their interaction with the vascular endothelium are believed to play a key role in acute arterial thrombosis (3). Platelet aggregation and adherence to the blood vessel are promoted by thromboxane generated by the platelet. Endothelially derived nitric oxide can inhibit platelet aggregation and adherence, while prostacyclin can inhibit aggregation (4).

The major limitation to the long-term use of aspirin is the increased risk of gastrointestinal bleeding, and development or exacerbation of gastric and duodenal ulcers. Kurata and Abbey (5) reported that a daily dose of $1000 \mathrm{mg}$ of aspirin increased the risk of hospitalization for gastric and duodenal ulcers by approximately eightfold. In a long-term study of every-otherday aspirin use $(325 \mathrm{mg}$ ) for prevention of cardiovascular diseases, the incidence of duodenal ulcers was significantly increased above that in the placebo group (6). Even with a dose of aspirin as low as $100 \mathrm{mg}$ per day, in a study of patients with coronary artery disease, the prevalence of gastric erosions was found to be increased (7).

Gastrointestinal ulceration is associated with the use of all nonsteroidal anti-inflammatory drugs (NSAIDs) ${ }^{1}$ and is directly related to the ability of these drugs to inhibit cyclo-oxygenase activity, thereby suppressing the formation of prostaglandins (8). We recently described a novel series of compounds that consist of an NSAID (e.g., flurbiprofen, diclofenac, ketoprofen ) linked to a nitric oxide-releasing moiety $(9,10)$. These "NONSAIDs" exhibit anti-inflammatory activities comparable to the parent NSAID, but have significantly reduced gastrointestinal toxicity (9-11). NSAIDs, unlike aspirin, are not used as anti-thrombotics, because they inhibit cyclo-oxygenase activity in a reversible manner. Thus, platelet thromboxane synthesis recovers when plasma levels of the NSAID decrease below those necessary for inhibition of cyclo-oxygenase.

Since the addition of a nitroxybutyl group to several NSAIDs resulted in a profound reduction in their ability to produce gastrointestinal injury, we proposed that a similar derivatization of aspirin may yield a compound with anti-thrombotic activity, but with greater tolerability in the gastrointestinal tract. In the present study we have compared aspirin to a nitric oxidereleasing aspirin derivative (NCX 4215; Fig. 1) in terms of their ability to inhibit platelet aggregation in vitro and ex vivo, their effects on prostaglandin and thromboxane synthesis and their ulcerogenic properties in the stomach.

\section{Methods}

Animals. Male, Wistar rats (175-200 grams) were obtained from Charles River Breeding Farms (Montreal, Quebec) and were housed in

1. Abbreviation used in this paper: NSAID, nonsteroidal anti-inflammatory drug. 


\section{Acetylsalicylic Acid (ASA)}<smiles>COc1ccccc1C(=O)O</smiles>

NCX 4215

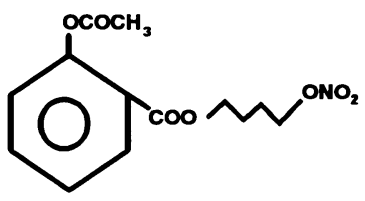

Figure 1. Structures of aspirin and NCX 4215 (acetylsalicylic acid 4(nitroxy)butylester).

polypropylene cages and fed standard laboratory chow and tap water ad libitum. All experimental protocols described in this report were approved by the Animal Care Committee of the University of Calgary.

Human platelet aggregation. Blood was collected from healthy volunteers $(n=6)$, who had not ingested aspirin or other nonsteroidal anti-inflammatory drugs for at least $14 \mathrm{~d}$ before donating blood, and washed platelets $\left(2 \times 10^{8}\right.$ platelets $\left./ \mathrm{ml}\right)$ were prepared as described by Radomski and Moncada (13). Aliquots $(0.4 \mathrm{ml})$ of the platelets were added into a cuvette of a dual channel platelet aggregometer (Payton Instruments, Buffalo, NY) and stirred continuously at $37^{\circ} \mathrm{C}$. Several concentrations of thrombin $(1-100 \mathrm{U} / \mathrm{ml})$ were tested for their ability to induce platelet aggregation. The concentration producing $85-95 \%$ maximal aggregation was selected for use in the remainder of the experiment. Aspirin or NCX 4215 was added to the platelet suspensions at concentrations in the $0.03-5 \mathrm{mM}$ range (always in a volume of $20 \mu \mathrm{l}$ ) and $10 \mathrm{~min}$ later the platelets were stimulated with thrombin. Aggregation was monitored for the ensuing $5 \mathrm{~min}$.

An additional series of experiments was performed in which platelets were preincubated with $10 \mu \mathrm{M}$ hemoglobin for 2 min before addition of aspirin $(100 \mu \mathrm{M}), \mathrm{NCX} 4215(100 \mu \mathrm{M})$ or vehicle. This concentration of hemoglobin has previously been shown to prevent nitric oxidemediated inhibition of platelet aggregation, while not significantly affecting thrombin-induced platelet aggregation (14). Hemoglobin binds to and inactivates nitric oxide (15). As a positive control in these experiments, the effects of incubation of the platelets in the presence of sodium nitroprusside $(100 \mu \mathrm{M})$, with or without hemoglobin, was also assessed.

Nitric oxide generation. Nitric oxide generation from NCX 4215 versus aspirin was examined using chemiluminescence detection of nitric oxide. Washed platelets were prepared as described above. Aliquots of the platelets $(0.4 \mathrm{ml})$ were incubated in the presence of aspirin $(1,10,100$, or $1000 \mu \mathrm{M}) \mathrm{NCX} 4215$ (same concentrations) or buffer for 1 or $10 \mathrm{~min}$. To establish whether NCX 4215 could spontaneously liberate nitric oxide, the drug $(1000 \mu \mathrm{M})$ was incubated at $37^{\circ} \mathrm{C}$ for 5 min with buffer in the absence of washed platelets. Incubations were terminated by centrifugation $(1000 \mathrm{~g} ; 5 \mathrm{~min})$ and the cell-free supernatant analysed for nitric oxide concentration, as described by Palmer et al. (16). Briefly, $100 \mu \mathrm{l}$ of the sample was injected into a reaction vessel containing the reducing solution consisting of glacial acetic acid $(5 \mathrm{ml})$ and potassium iodide $(100 \mu \mathrm{l}$ of $100 \mu \mathrm{M})$. Nitrite present in the injected buffer was reduced under these conditions to nitric oxide and the latter stripped from the reducing mixture by bubbling of helium. Nitric oxide generated was carried by the flow of helium to the nitric oxide analyzer, where it reacted with ozone, generated internally by electostatic discharge, and the light emitted measured. The amounts of nitric oxide detected in $\mathrm{mV}$ were convered to $\mathrm{nM}$ concentrations using

a nitrite standard curve. These experiments were performed on three separate preparations of platelets, each time in triplicate.

Platelet $c G M P$ and $c A M P$ concentrations. Washed platelets were prepared from human blood, as above, and aliquots $(0.4 \mathrm{ml})$ were added to Eppendorf tubes and placed in a shaking water bath $\left(37^{\circ} \mathrm{C}\right)$. Aspirin $(1,10,100$, or $1000 \mu \mathrm{M}), \mathrm{NCX} 4215$ (same concentrations) or vehicle (methanol) was then added to the platelets as a total volume of $20 \mu \mathrm{l}$. One or ten min later the tubes were centrifuged at $1000 \mathrm{~g}$ for $20 \mathrm{~s}$, then the supernatant was removed. Ice cold phosphate buffer $(\mathrm{pH} 7.4 ; 100$ $\mu 1$ ) was added to the pellet and the pellet was then sonicated for $20 \mathrm{~s}$. The samples were stored at $-20^{\circ} \mathrm{C}$ until the assays for cGMP and cAMP were performed, using specific enzyme-linked immunosorbent assays. As a positive control, the effects of incubation of the platelets in the presence of sodium nitroprusside $(1-1000 \mu \mathrm{M})$ were also assessed. These experiments were performed on at least three separate preparations of platelets, each time in triplicate.

Rat platelet aggregation. The effects of aspirin and NCX 4215 on rat platelet aggregation were studied ex vivo. Rats were given vehicle, aspirin ( $100 \mathrm{mg} / \mathrm{kg}$ ), or an equimolar dose of NCX 4215 (166 mg/ $\mathrm{kg}$ ) or sodium salicylate $(89 \mathrm{mg} / \mathrm{kg})$ and three hours later were anesthetized with ether. Blood was collected from the descending aorta and platelet-rich plasma (PRP) was prepared (13). Aliquots $(0.5 \mathrm{ml})$ of the PRP were placed in the cuvette of a Payton platelet aggregometer and continuously stirred at $37^{\circ} \mathrm{C}$. After $1 \mathrm{~min}$, adenosine diphosphate (ADP) was added into the cuvette, and aggregation was monitored for the following $5 \mathrm{~min}$. ADP was tested at concentrations in the $2.5-40 \mu \mathrm{M}$ range. The responsiveness of platelets from these rats to collagen was also assessed in the same manner. A concentration of collagen (25$100 \mu \mathrm{g} / \mathrm{ml}$ ) causing 85-95\% maximal aggregation was used in each assay. For comparison to a nitric oxide donor, the effects of orally administered sodium nitroprusside ( $145 \mathrm{mg} / \mathrm{kg}$; equimolar to the 166 $\mathrm{mg} / \mathrm{kg}$ dose of NCX 4215) on ADP- and collagen-induced platelet aggregation were also assessed.

The effects of aspirin $(100 \mathrm{mg} / \mathrm{kg})$ and NCX $4215(166 \mathrm{mg} / \mathrm{kg})$ on platelet aggregation induced by thrombin were assessed, at 1, 3, 6, and $12 \mathrm{~h}$ after administration of the test drugs ( $n=4-6$ per group). Various concentrations of thrombin $(1-100 \mathrm{U} / \mathrm{ml})$ were tested for their ability to induce aggregation of platelets from vehicle-treated rats, and the concentration causing 85-95\% maximal aggregation was used in the experiments with platelets from aspirin- or NCX 4125-treated rats. Results were then expressed as percent inhibition of aggregation relative to platelets from vehicle-treated rats.

Thromboxane and prostaglandin synthesis. The synthesis of thromboxane by whole blood was determined using the method of Patrono et al. (17). Groups of rats ( $n=5$ each) were given ASA $(10-100 \mathrm{mg} /$ $\mathrm{kg}$ ), NCX $4215(30-300 \mathrm{mg} / \mathrm{kg}$ ) or vehicle orally and were anesthetized with ether $3 \mathrm{~h}$ later. Blood was drawn from the descending aorta into a syringe and immediately $1 \mathrm{ml}$ of the blood was transferred to a glass tube. The tubes of blood were allowed to stand at $37^{\circ} \mathrm{C}$ for 45 min, after which they were centrifuged $(1000 \mathrm{~g} ; 10 \mathrm{~min})$. The serum was transferred to Eppendorf tubes then frozen at $-20^{\circ} \mathrm{C}$. Thromboxane $\mathrm{B}_{2}$ concentrations in the serum samples were measured using a specific enzyme-linked immunosorbent assay. An additional group of rats ( $n$ $=5$ ) received NCX 4215 ( $100 \mathrm{mg} / \mathrm{kg}$ ) intraperitoneally, and $3 \mathrm{~h}$ later blood was collected for determination of thromboxane synthesis, as above.

A separate series of experiments was performed to determine the effects of daily administration of aspirin $(100 \mathrm{mg} / \mathrm{kg})$ or an equimolar dose of NCX 4215 ( $166 \mathrm{mg} / \mathrm{kg}$ ) for five days on thromboxane synthesis. Three hours after the final (fifth) dose of the drugs or vehicle, blood was collected and processed, as described above, for measurement of thromboxane $\mathbf{B}_{2}$ synthesis.

To determine the extent of any inhibitory actions of NCX 4215 versus aspirin on gastric prostaglandin synthesis, a series of studies were performed in which the capacity of this tissue to generate 6-keto prostaglandin $\mathrm{F}_{1_{\alpha}}$ (the stable hydration product of prostacyclin) in vitro was determined. Three rats were deprived of food but not water overnight. They were then killed and the stomach removed. The corpus 
region of the stomach from each rat was then divided into approximately equal sized pieces $(\sim 100 \mathrm{mg})$ and randomly added to tubes containing $1 \mathrm{ml}$ of sodium phosphate buffer ( $\mathrm{pH} \mathrm{7.4)}$ alone, or containing aspirin $1,10,100$, or $1000 \mu \mathrm{M}$ ) or NCX 4215 (same concentrations). The tissue samples were minced with scissors for $10 \mathrm{~s}$, then the tubes were placed in a shaking water bath $\left(37^{\circ} \mathrm{C}\right)$ for $10 \mathrm{~min}$. After centrifugation $(9000 \mathrm{~g})$ for $3 \mathrm{~min}$, the supernatants were frozen on dry ice then stored at $-70^{\circ} \mathrm{C}$ until an ELISA assay was performed to determine concentrations of 6-keto PGF P. $_{1}$.

Gastric mucosal damage. Groups of rats $(n=5$ each) were deprived of food, but not water, for $18 \mathrm{~h}$ and were then given aspirin (10-120 $\mathrm{mg} / \mathrm{kg}), \mathrm{NCX} 4215(30-300 \mathrm{mg} / \mathrm{kg}$ ) or vehicle orally. The volume of drug/vehicle administered was $1 \mathrm{ml} / \mathrm{kg}$. Three hours later the rats were killed and the stomach excised. An observer unaware of the treatment the rats had received scored the severity of macroscopically visible damage using a method described previously (18). This method involves measuring the length of lesions in $\mathrm{mm}$, then summing the lengths of all lesions observed in each stomach. After scoring the gastric tissue was fixed in neutral buffered formalin and processed by routine techniques prior to embedding in paraffin, sectioning and staining with hematoxylin and eosin. The slides were coded to avoid observer bias prior to being examined under a light microscope.

An additional series of experiments examined the effects of aspirin and NCX 4215 on the stomach after repeated administration over a twoweek period. Groups of five rats each received vehicle, aspirin (100 $\mathrm{mg} / \mathrm{kg}$ ) or NCX $4215(166 \mathrm{mg} / \mathrm{kg}$ ) orally each day. Three hours following the final dose (on day 14), the rats were killed, the stomach examined and the severity of damage scored as described above.

Plasma salicylate levels. To determine if NCX 4215 was metabolized to salicylate, a series of experiments were performed in which groups of four rats each were anesthetized with ether $1,3,6$, or $12 \mathrm{~h}$ after oral administration of aspirin $(100 \mathrm{mg} / \mathrm{kg})$ or an equimolar dose of NCX $4215(166 \mathrm{mg} / \mathrm{kg})$. Plasma salicylate concentrations were determined spectrophotometrically (19) using a commercially available kit. A standard curve was constructed using plasma spiked with sodium salicylate in the $10-1000 \mu \mathrm{g} / \mathrm{ml}$ range, and similar concentrations of aspirin and NCX 4215 were also tested in the assay. The assay did not detect aspirin or NCX, while the detection of salicylate was linear over the range of concentrations tested $\left(r^{2}=0.98 ; n=6\right)$.

Systemic arterial blood pressure. Rats were anesthetized with urethane (25\%; $0.6 \mathrm{ml} / 100$ grams body weight, i.p.) and a carotid artery was cannulated for measurement of systemic arterial blood pressure. After recording pressure for $15 \mathrm{~min}$, vehicle, aspirin $(100 \mathrm{mg} / \mathrm{kg})$ or an equimolar dose of NCX $4215(166 \mathrm{mg} / \mathrm{kg})$ or sodium nitroprusside $(145 \mathrm{mg} / \mathrm{kg})$ was injected intravenously. Blood pressure was continuously recorded for the ensuing three hours. Each group consisted of five rats.

Materials. The assay kits for measurement of $\mathrm{TXB}_{2}, 6-k e t o \mathrm{PGF}_{1 \alpha}$, cyclic GMP and cyclic AMP were obtained from Cayman Chemical Company, Ann Arbor, MI. NCX 4215 (acetylsalicylic acid 4-(nitroxy)butylester) was provided by NicOx Ltd., London, England. Adenosine diphosphate, rabbit hemoglobin, sodium nitroprusside, human thrombin and the assay kit for plasma salicylate were obtained from Sigma Chemical Co. (St. Louis, MO). Type 6 collagen was obtained from Hormon Chemie, Munich, Germany. Carboxymethylcellulose was obtained from British Drug House Ltd. (Poole, England). All other reagents were obtained from Fisher Scientific Ltd., Edmonton, Alberta.

Statistical analysis. All data are expressed as the mean \pm SE. Comparisons of two groups of data were performed using the Student's $t$ test, whereas comparisons among more than two groups of data were performed using a one way analysis of variance followed by a StudentNewman-Keuls post-hoc test (Instat software package, GraphPad Software, San Diego, CA). With all such analyses, an associated probability $(P$ value $)$ of $<5 \%$ was considered significant.

\section{Results}

In vitro human platelet studies. Addition of aspirin to human platelets resulted in concentration-dependent inhibition of

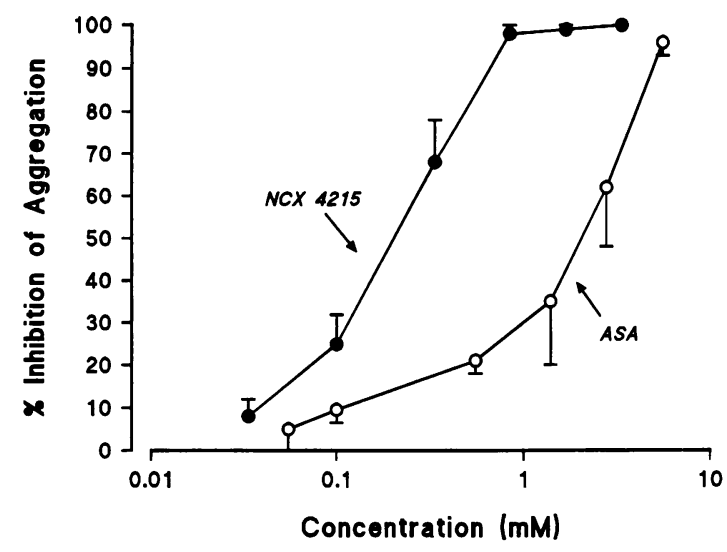

Figure 2. Effects of aspirin and NCX 4215 on thrombin-induced human platelet aggregation in vitro. Each point represents the mean $\pm S E$ of six experiments. The platelets were incubated with the test drugs for 10 min before stimulation with thrombin, and aggregation was monitored for $5 \mathrm{~min}$ thereafter. Results are expressed as a percent inhibition of aggregation relative to the platelets pretreated with the vehicle.

thrombin-induced aggregation (Fig. 2). NCX 4215 also concentration-dependently inhibited platelet aggregation, but this compound was more potent in this action than aspirin. Indeed, the $\mathrm{IC}_{50}$ for NCX $4215(0.34 \mathrm{mM})$ was about one-seventh that for aspirin $(2.24 \mathrm{mM})$.

Chemiluminescence detection of nitric oxide concentrations demonstrated that incubation of platelets with aspirin at concentrations of $1-1000 \mu \mathrm{M}$ did not result in significant liberation of nitric oxide (Fig. 3). However, incubation of platelets with NCX 4215 at concentrations of 100 and $1000 \mu \mathrm{M}$ resulted in significant liberation of nitric oxide. This release was apparent after only $1 \mathrm{~min}$ of incubation of the drug with platelets. While

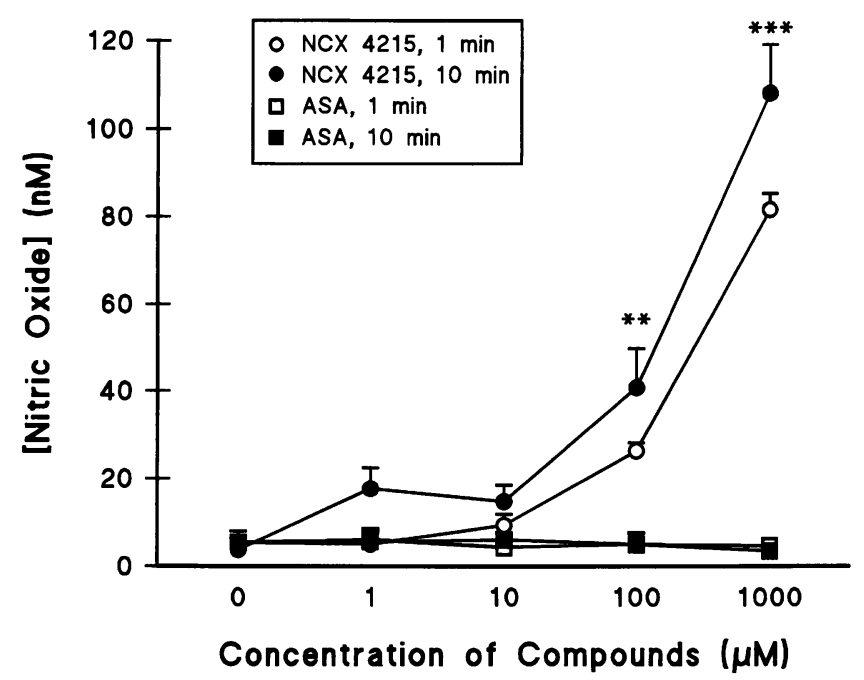

Figure 3. Nitric oxide release, determined by chemiluminescence, from aspirin and NCX 4215 incubated with human platelets. Significant amounts of nitric oxide were released from NCX 4215 at concentrations of 100 and $1000 \mu \mathrm{M}$ when incubated with the platelets for 1 or $10 \mathrm{~min}$ ( ${ }^{* *} P<0.01, * * * P<0.001$ : NCX 4215-treated platelets [both incubation times] versus the platelets treated with vehicle). Nitric oxide release was not detectable when NCX 4215 was incubated in the absence of platelets. 
Table I. Effects of Aspirin, NCX 4215, and Sodium Nitroprusside on Platelet cAMP and cGMP Levels

\begin{tabular}{|c|c|c|c|}
\hline Group & $\begin{array}{l}\text { Incubation } \\
\text { Time }\end{array}$ & cAMP & cGMP \\
\hline & $\min$ & $p g / m l$ & $p g / m l$ \\
\hline Vehicle & 1 & $74.5 \pm 14.5$ & $5.44 \pm 0.98$ \\
\hline $\operatorname{ASA}(1 \mu \mathrm{M})$ & 1 & $58.8 \pm 9.0$ & $7.41 \pm 2.58$ \\
\hline ASA $(10 \mu \mathrm{M})$ & 1 & $57.1 \pm 6.6$ & $5.42 \pm 0.91$ \\
\hline ASA $(100 \mu \mathrm{M})$ & 1 & $47.8 \pm 3.1$ & $6.80 \pm 0.41$ \\
\hline ASA $(1 \mathrm{mM})$ & 1 & $157.2 \pm 21.5^{\S}$ & $10.18 \pm 1.58$ \\
\hline NCX-4215 $(1 \mu \mathrm{M})$ & 1 & $56.3 \pm 11.6$ & $12.93 \pm 6.63$ \\
\hline $\operatorname{NCX}-4215(10 \mu \mathrm{m})$ & 1 & $73.2 \pm 29.5$ & $6.24 \pm 2.19$ \\
\hline NCX-4215 $(100 \mu \mathrm{M})$ & 1 & $66.5 \pm 14.4$ & $34.13 \pm 17.73$ \\
\hline NCX-4215 (1 mM) & 1 & $150.3 \pm 17.5^{*}$ & $55.91 \pm 42.07$ \\
\hline Nitroprusside $(1 \mu \mathrm{M})$ & 1 & $95.2 \pm 3.2$ & $5.51 \pm 0.32$ \\
\hline Nitroprusside $(10 \mu \mathrm{M})$ & 1 & $94.1 \pm 10.0$ & $9.77 \pm 2.98$ \\
\hline Nitroprusside $(100 \mu \mathrm{M})$ & 1 & $57.5 \pm 15.1$ & $10.80 \pm 1.96$ \\
\hline Nitroprusside (1 mM) & 1 & $197.6 \pm 3.0^{8}$ & $42.71 \pm 9.02$ \\
\hline Vehicle & 10 & $56.0 \pm 16.6$ & $5.09 \pm 1.08$ \\
\hline ASA $(1 \mu \mathrm{M})$ & 10 & $58.9 \pm 7.8$ & $6.05 \pm 0.81$ \\
\hline ASA $(10 \mu \mathrm{m})$ & 10 & $82.2 \pm 3.2$ & $6.47 \pm 1.41$ \\
\hline ASA $(100 \mu \mathrm{M})$ & 10 & $71.8 \pm 21.7$ & $5.66 \pm 1.36$ \\
\hline ASA $(1 \mathrm{mM})$ & 10 & $111.8 \pm 16.9$ & $13.16 \pm 3.24$ \\
\hline NCX-4215 $(1 \mu \mathrm{M})$ & 10 & $56.3 \pm 11.6$ & $6.17 \pm 1.07$ \\
\hline NCX-4215 $(10 \mu \mathrm{M})$ & 10 & $73.2 \pm 29.5$ & $17.21 \pm 10.06$ \\
\hline NCX-4215 $(100 \mu \mathrm{M})$ & 10 & $66.5 \pm 14.4$ & $45.54 \pm 4.28^{\ddagger}$ \\
\hline NCX-4215 (1 mM) & 10 & $150.3 \pm 17.5^{*}$ & $77.77 \pm 5.88^{\S}$ \\
\hline Nitroprusside $(1 \mu \mathrm{M})$ & 10 & $37.8 \pm 2.6$ & $7.6 \pm 2.23$ \\
\hline Nitroprusside $(10 \mu \mathrm{M})$ & 10 & $55.2 \pm 13.0$ & $42.89 \pm 2.81 *$ \\
\hline Nitroprusside $(100 \mu \mathrm{M})$ & 10 & $46.3 \pm 11.8$ & $156.30 \pm 4.41^{\S}$ \\
\hline Nitroprusside (1 mM) & 10 & $161.8 \pm 6.1^{8}$ & $370.18 \pm 32.32^{\S}$ \\
\hline
\end{tabular}

${ }^{*} P<0.05,{ }^{\ddagger} P<0.01,{ }^{\S} P<0.001$ compared with the corresponding vehicle-treated group (ANOVA and Student-Newman-Keuls Multiple Comparison Test). Results are expressed as mean \pm SE $(n=3-6$ per group).

liberation of nitric oxide from this compound occurred when the drug was incubated with platelets, nitric oxide release was not detectable from samples of the drug incubated with buffer alone.

Exposure of human platelets to aspirin at concentrations of 1 to $1000 \mu \mathrm{M}$ did not significantly affect intracellular cyclic GMP levels during the first minute of exposure (Table 1). Cyclic AMP levels were unaffected during this period by exposure to any of the test drugs at concentrations in the 1 to $100 \mu \mathrm{M}$ range. However, all three test drugs, at a $1 \mathrm{mM}$ concentration, significantly elevated platelet cAMP levels. When exposure to the test drugs was extended to $10 \mathrm{~min}$, aspirin did not exert any significant effect on cGMP or cAMP levels. However, both NCX 4215 (100 and $1000 \mu \mathrm{M})$ and sodium nitroprusside (10, 100 , and $1000 \mu \mathrm{M})$ markedly elevated cGMP levels. The elevations in platelet cGMP levels were substantially greater with sodium nitroprusside than with NCX 4215. This corresponds to enhanced anti-aggregatory effects of sodium nitroprusside versus NCX 4215 on thrombin-induced platelet aggregation. At concentrations of 10 and $100 \mu \mathrm{M}, \mathrm{NCX} 4215$ inhibited thrombin-induced platelet aggregation by $2 \pm 1 \%$ and $25 \pm 7 \%$, respectively, while the same concentrations of sodium nitroprusside

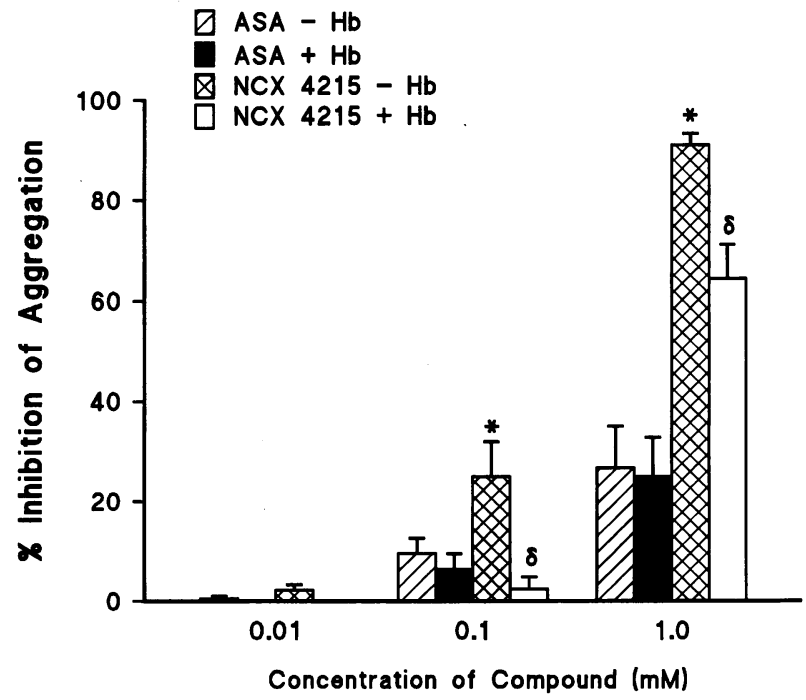

Figure 4. Effects of preincubation of human platelets with $10 \mu \mathrm{M}$ hemoglobin on anti-aggregatory effects of aspirin and NCX 4215. Plateletrich plasma was preincubated with hemoglobin for $2 \mathrm{~min}$, then incubated with aspirin (ASA), NCX 4215 or vehicle for $10 \mathrm{~min}(n=6)$. The platelets were then stimulated with thrombin and aggregation was monitored for $5 \mathrm{~min}$. Results are expressed as percent inhibition of aggregation relative to the platelets pretreated with the vehicle but not hemoglobin. Hemoglobin did not significantly affect the extent of thrombininduced aggregation in platelets pretreated with vehicle or in platelets pretreated with aspirin. However, hemoglobin did significantly reduce the anti-aggregatory effects of NCX $4215\left(^{*} P<0.05\right.$ compared to that aspirin group not treated with hemoglobin; ${ }^{\delta} P<0.05$ compared with the corresponding group not treated with hemoglobin.

inhibited thrombin-induced platelet aggregation by $40 \pm 4 \%$ and $63 \pm 1 \%$, respectively.

Hemoglobin did not significantly affect the extent of platelet aggregation observed after stimulation with thrombin, as reported previously (13). Similarly, hemoglobin did not signifi-

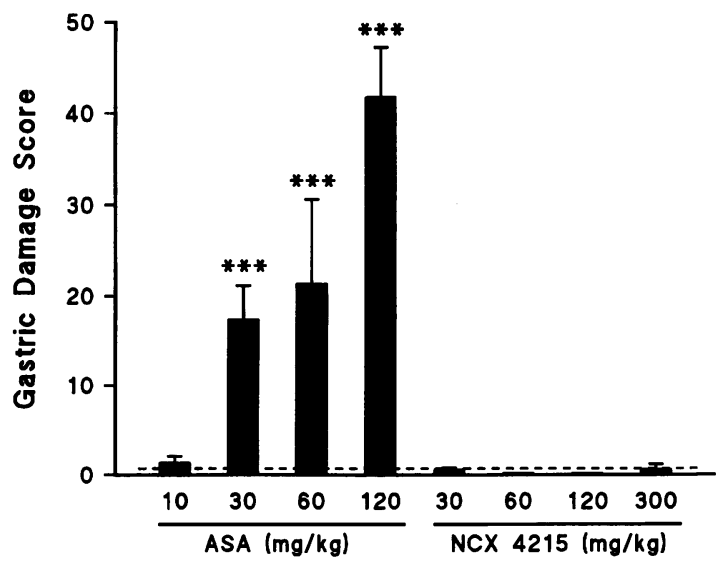

Figure 5. Effects of orally administered aspirin (ASA) and NCX 4215 on macroscopically assessed gastric damage severity. The dotted line shows the mean gastric damage score in vehicle-treated rats. Each group consisted of five rats. Damage was assessed three hours after oral administration of the compounds by an observer unaware of the treatments the rats had received. Asterisks denote a significant $(P<0.001)$ difference compared to the vehicle-treated group. 


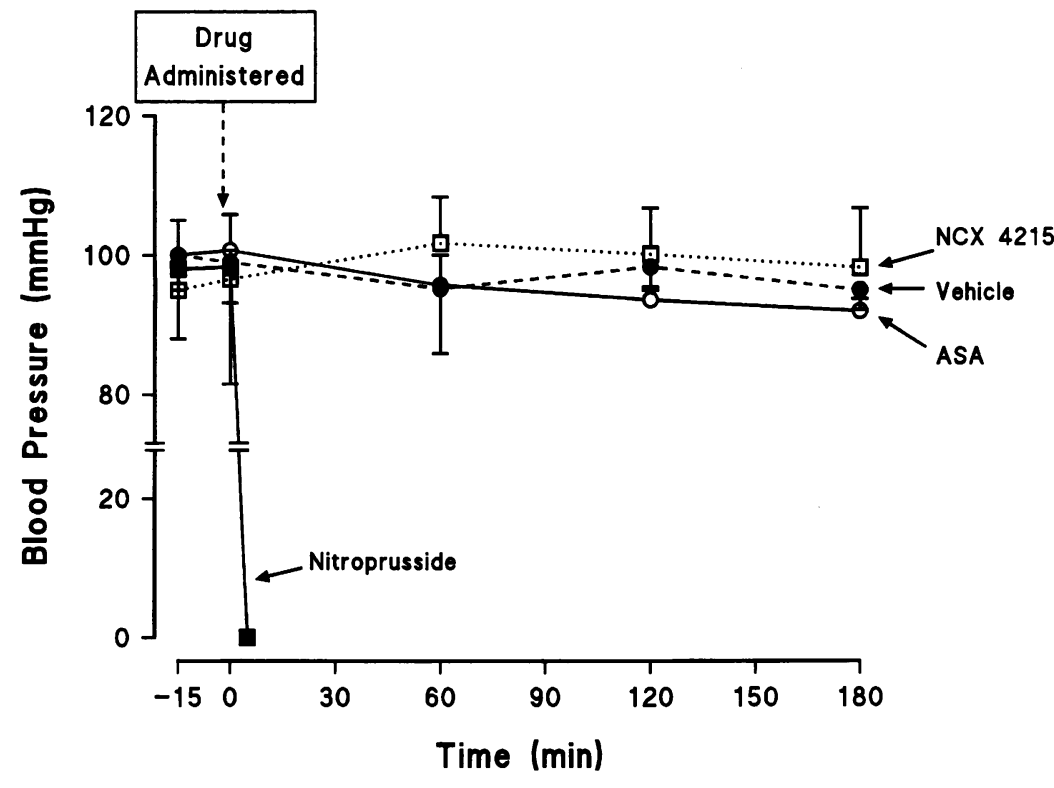

Figure 6. Effects of intravenous administration of aspirin $(100 \mathrm{mg} / \mathrm{kg}$ ) or equimolar doses of NCX 4215 (166 mg/ $\mathrm{kg}$ ) or sodium nitroprusside ( $145 \mathrm{mg} / \mathrm{kg}$ ) on systemic arterial blood pressure in anesthetized rats. All rats receiving intravenous sodium nitroprusside died within $5 \mathrm{~min}$ of its administration. Aspirin and NCX 4215 did not significantly affect systemic arterial blood pressure relative to the vehicle-treated group. cantly affect the inhibition of platelet aggregation induced by aspirin at concentration of 10 to $1000 \mu \mathrm{M}$ (Fig. 4). However, hemoglobin significantly reduced the anti-aggregatory activity of the NCX 4215 (100 and $1000 \mu \mathrm{M})$. Hemoglobin also significantly attenuated the anti-aggregatory effect of sodium nitroprusside $(100 \mu \mathrm{M})$, reducing its inhibition of thrombin-stimulated aggregation from $63 \pm 1 \%$ to $33 \pm 3 \%(P<0.01)$.

In vivo rat studies: gastric damage. Oral administration of aspirin resulted in the formation of extensive hemorrhagic lesions in the stomach. These lesions were usually linear, running along the crests of rugal folds, and were observed primarily in the corpus region. Histologically, the lesions were found to be confined to the mucosal layer, not penetrating the muscularis mucosae. The severity of gastric damage induced by aspirin increased in a dose-dependent manner (Fig. 5). In contrast, NCX 4215 did not produce significant gastric damage at any of the doses tested $(30-300 \mathrm{mg} / \mathrm{kg}$ ). The absence of damage following NCX 4215 administration was confirmed histologically.

Similar differences in gastric damage were observed in the study in which rats were killed various times after drug administration. In rats killed 1, 3, 6 and $12 \mathrm{~h}$ after administration of aspirin ( $100 \mathrm{mg} / \mathrm{kg} ; n=4$ per group), the mean damage scores were: $50.5 \pm 4.6,54.5 \pm 11.3,62.0 \pm 15.6$ and $42.3 \pm 16.8$. Damage was not observed in rats examined at the same times after administration of an equimolar dose of NCX $4215(P<0.01$ at all time points). Moreover, following daily administration of these doses of aspirin and NCX 4215 for $14 \mathrm{~d}$, there was a clear difference between the drugs in terms of gastric damage. In the aspirin-treated rats, there was evidence of persistent hemorrhagic lesions and scar tissue where lesions had healed. Histologically the lesions involved the full depth of the mucosa. The mean damage score was $26.4 \pm 0.9(n=5)$. However, in the rats treated with NCX $4215(n=5)$, damage was not detectable, either macroscopically or histologically.

In vivo rat studies: blood pressure. Intravenous administration of aspirin $(100 \mathrm{mg} / \mathrm{kg})$ or an equimolar dose of NCX 4215 $(166 \mathrm{mg} / \mathrm{kg})$ to anesthetized rats did not significantly affect systemic arterial blood pressure, which was monitored for the ensuing $3 \mathrm{~h}$ (Fig. 6). However, administration of an equimolar dose of sodium nitroprusside $(145 \mathrm{mg} / \mathrm{kg}$ ) led within $5 \mathrm{~min}$ to extensive hypotension and death.

In vivo rat studies: plasma salicylate levels. Oral administration of aspirin $(100 \mathrm{mg} / \mathrm{kg})$ resulted in the appearance of salicylate in plasma within one hour (Fig. 7). The levels of salicylate in plasma reached a peak at $3 \mathrm{~h}$ post-administration $(\sim 150 \mu \mathrm{g} / \mathrm{ml})$, remained at a similar level at 6 hours postadministration, and had declined to $\sim 60 \mu \mathrm{g} / \mathrm{ml}$ by $12 \mathrm{~h}$ after administration. Oral administration of an equimolar dose of NCX 4215 also resulted in the appearance of salicylate in plasma, but it was not detectable until $3 \mathrm{~h}$ after administration and was virtually all eliminated by $12 \mathrm{~h}$ after administration. Evaluation of the areas under the curves shown in Fig. 7 revealed that the amount of salicylate generated from NCX 4215 was $\sim 27 \%$ of that generated by an equimolar dose of aspirin. Neither aspirin nor NCX 4215 could be detected in this assay at concentrations in the $10-1000 \mu \mathrm{g} / \mathrm{ml}$ range.

Ex vivo rat studies: platelet aggregation. Aspirin and NCX 4215 inhibited ex vivo platelet aggregation induced by thrombin (Fig. 7). Platelets from rats treated $1-3 \mathrm{~h}$ earlier with aspirin $(100 \mathrm{mg} / \mathrm{kg})$ or an equimolar dose of NCX 4215 were significantly less responsive to thrombin than vehicle-treated controls. At 6 hours post-administration, the NCX 4215 continued to significantly inhibit thrombin-induced aggregation, although the effect was much more variable than at the earlier time points. At $12 \mathrm{~h}$ post-administration, neither NCX 4215 nor aspirin significantly inhibited aggregation.

Oral administration of aspirin $(100 \mathrm{mg} / \mathrm{kg})$ also significantly depressed the responsiveness of platelets, harvested 3 hours later, to stimulation by ADP or collagen (Fig. 8). Oral administration of an equimolar dose of sodium salicylate did not significantly affect platelet aggregation responses to ADP or collagen, when compared to platelets from vehicle-treated rats. However, platelets from rats treated orally with NCX 4215 were significantly less responsive to both ADP and collagen. In fact, the effects of NCX 4215 on platelet aggregation were not significantly different from those of aspirin. When platelets were harvested from rats $12 \mathrm{~h}$ after administration of the test 

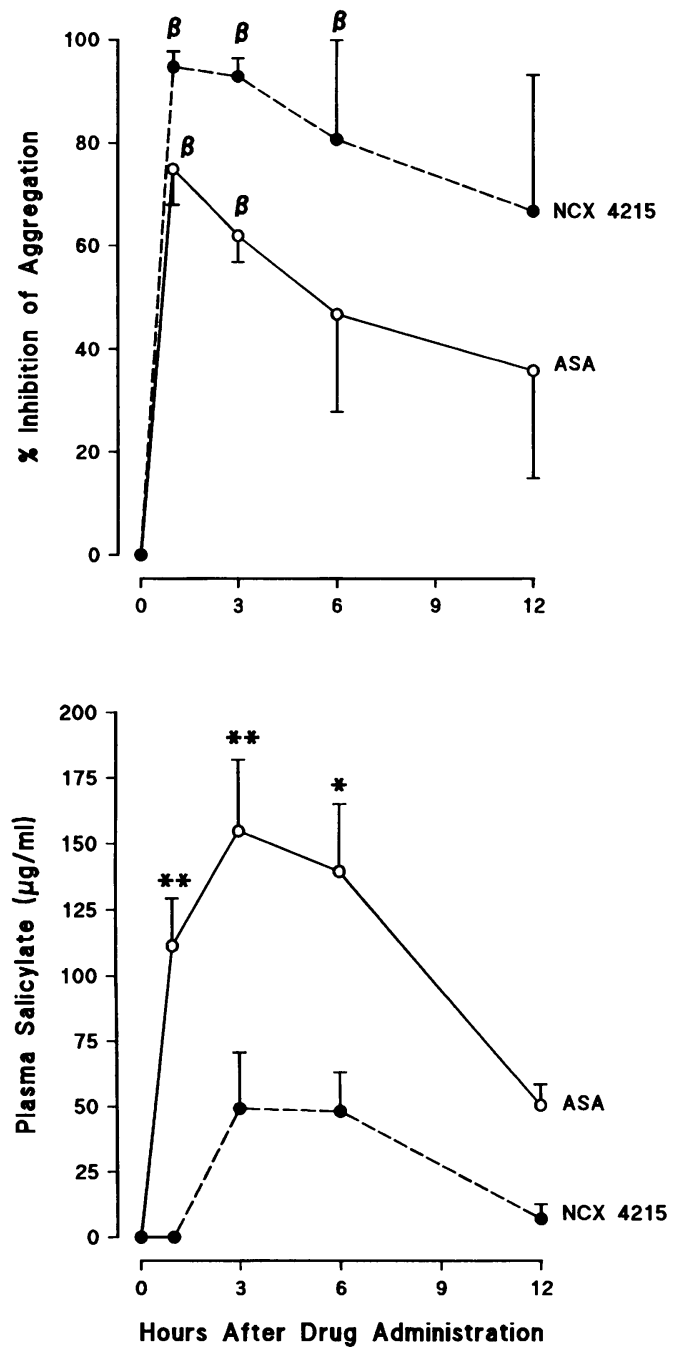

Figure 7. (Upper panel) Inhibition of thrombin-induced platelet aggregation by aspirin and NCX 4215 . Rats received aspirin $(100 \mathrm{mg} / \mathrm{kg})$ or an equimolar dose of NCX $4215(166 \mathrm{mg} / \mathrm{kg})$ orally and blood was collected $1-12 \mathrm{~h}$ later. Platelet aggregation in response to thrombin was assessed (see Methods) and the results expressed as percent inhibition of aggregation relative to that seen in platelets harvested from rats treated with the vehicle. ${ }^{\beta} P<0.05$ compared to vehicle-treated controls. Each point represents the mean $\pm S E$ for 4-6 rats. (Lower panel) Plasma salicylate levels up to $12 \mathrm{~h}$ following oral administration of aspirin ( 100 $\mathrm{mg} / \mathrm{kg})$ or an equimolar dose of NCX $4215(166 \mathrm{mg} / \mathrm{kg}) .(* P<0.05$, $* * P<0.01$ compared with the NCX 4215 group). Each point represents the mean $\pm \mathrm{SE}$ for four rats.

drugs, aspirin continued to completely inhibit the aggregation induced by collagen, while NCX 4215 no longer exhibited any effect.

When administered orally at an equimolar dose to that of NCX 4215 in the studies described above, sodium nitroprusside had no significant effect on platelet aggregation induced by ADP or collagen. All of the rats treated with sodium nitroprusside at this dose $(145 \mathrm{mg} / \mathrm{kg} ; n=3)$ were lethargic and in a state of semi-consciousness within $30 \mathrm{~min}$ of its administration. At the time of drawing the blood samples, these rats exhibited profound engorgement of the mesenteric circulation.

Ex vivo rat studies: thromboxane and prostaglandin synthesis. Oral administration of aspirin resulted in a dose-dependent
Vehicle

Salicylate

$\square$ ASA

$\triangle \operatorname{NCX} 4215$

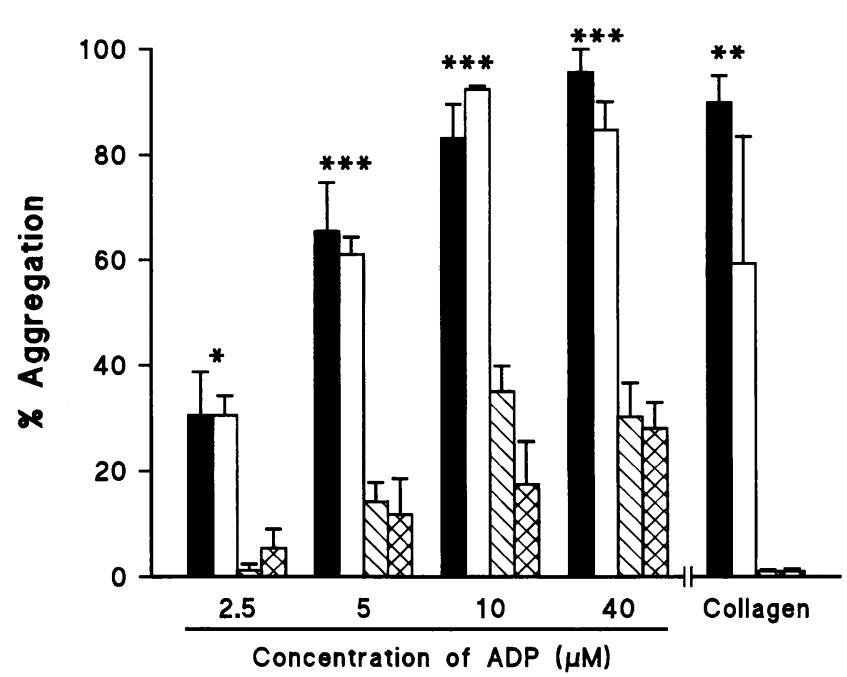

Figure 8. Effects of orally administered aspirin, salicylate, NCX 4215 or vehicle on platelet aggregation in response to stimulation with ADP or collagen. Platelets were collected from rats $3 \mathrm{~h}$ after administration of the drugs. Aspirin was given at a dose of $100 \mathrm{mg} / \mathrm{kg}$, and the other test drugs were given at doses equimolar to the aspirin dose. Asterisks denote significant inhibitory effects of aspirin and NCX 4215 relative to the other two groups $(* P<0.05, * * P<0.01, * * * P<0.001$ ). Each bar represents the mean $\pm \mathrm{SE}$ for at least five rats per group.

suppression of thromboxane synthesis by whole blood, with the $100 \mathrm{mg} / \mathrm{kg}$ dose inhibiting by $99 \%$ (Fig. 9). NCX 4215, at doses of 30 to $300 \mathrm{mg} / \mathrm{kg}$, was without significant effect on thromboxane synthesis. NCX $4215(100 \mathrm{mg} / \mathrm{kg})$ also had no significant effect on thromboxane synthesis when administered

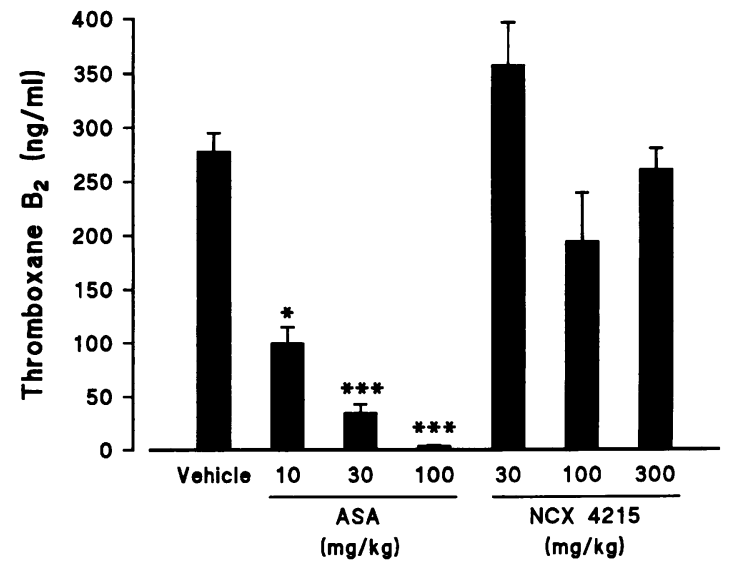

Figure 9. Effects of orally administered aspirin or NCX 4215 on thromboxane synthesis by rat blood. Blood was collected $3 \mathrm{~h}$ after administration of the test drugs, or vehicle. Production of thromboxane $\mathbf{B}_{2}$ by whole blood was measured at the end of a 45-min incubation period. While aspirin significantly inhibited thromboxane synthesis $(* P<0.05$, $* * * P<0.001$ ), NCX 4215 had no effect. Each bar represents the mean $\pm \mathrm{SE}$ for five rats per group. 
Table II. Effects of Daily Administration of Aspirin or NCX 4215 for $5 \mathrm{~d}$ on Thromboxane Synthesis by Rat Whole Blood

\begin{tabular}{lc}
\hline \multicolumn{1}{c}{ Treatment } & Thromboxane $B_{2}(\mathrm{ng} / \mathrm{ml})$ \\
\hline Vehicle & $459 \pm 28$ \\
Aspirin $(20 \mathrm{mg} / \mathrm{kg})$ & $17 \pm 3^{*}$ \\
NCX $4215(33 \mathrm{mg} / \mathrm{kg})$ & $505 \pm 23$ \\
Aspirin $(50 \mathrm{mg} / \mathrm{kg})$ & $6 \pm 1^{*}$ \\
NCX $4215(83 \mathrm{mg} / \mathrm{kg})$ & $398 \pm 22$
\end{tabular}

Production of thromboxane $\mathbf{B}_{2}$ by whole blood was measured at the end of a 45 -min incubation period. $* P<0.01$ compared with the vehicletreated group.

intraperitoneally (mean $\mathrm{TXB}_{2}$ synthesis of $310 \pm 14 \mathrm{ng} / \mathrm{ml}$, versus $278 \pm 20 \mathrm{ng} / \mathrm{ml}$ in controls ).

Daily administration of aspirin for $5 \mathrm{~d}$ at doses of 20 and 50 $\mathrm{mg} / \mathrm{kg}$ resulted in near-complete suppression of thromboxane synthesis by whole blood (Table II). However, administration of equimolar doses of NCX 4215 was without significant effect on thromboxane synthesis.

As shown in Table III, incubation of gastric tissue in the presence of aspirin resulted in a concentration-dependent (1$1000 \mu \mathrm{g} / \mathrm{ml}$ ) inhibition of 6-keto prostaglandin $F_{1 \times}$ synthesis. However, equimolar concentrations of NCX 4215 had no significant effect on gastric prostaglandin synthesis.

\section{Discussion}

The anti-thrombotic actions of aspirin are related to its ability to suppress thromboxane synthesis, by inhibiting the enzyme cyclo-oxygenase in platelets (2). However, suppression of cyclo-oxygenase is associated with significant toxicity in the gastrointestinal tract (20) and, less frequently, in the kidney (21). This toxicity limits the utility of aspirin for long-term use as an anti-thrombotic agent. In this study we have tested the hypothesis that addition of a nitric oxide-releasing moiety to aspirin would yield a compound that retained its anti-thrombotic actions, but had reduced gastrointestinal toxicity. Our initial

Table III. Effects of Aspirin and NCX 4215 on Gastric Prostaglandin Syntheses in Vitro

\begin{tabular}{lc}
\hline \multicolumn{1}{c}{ Treatment } & 6-keto Prostaglandin $\mathrm{F}_{1 \times}(\mathrm{pg} / \mathrm{mg})$ \\
\hline Vehicle & $1121 \pm 141$ \\
Aspirin $(1 \mu \mathrm{g} / \mathrm{ml})$ & $1180 \pm 193$ \\
NCX $4215(1.66 \mu \mathrm{g} / \mathrm{ml})$ & $1321 \pm 214$ \\
Aspirin $(10 \mu \mathrm{g} / \mathrm{ml})$ & $558 \pm 218^{\ddagger}$ \\
NCX $4215(16.6 \mu \mathrm{g} / \mathrm{ml})$ & $1451 \pm 229$ \\
Aspirin $(100 \mu \mathrm{g} / \mathrm{ml})$ & $531 \pm 92^{* \ddagger}$ \\
NCX $4215(166 \mu \mathrm{g} / \mathrm{ml})$ & $1202 \pm 212$ \\
Aspirin $(1000 \mu \mathrm{g} / \mathrm{ml})$ & $145 \pm 23^{* \ddagger}$ \\
NCX 4215 $(1660 \mu \mathrm{g} / \mathrm{ml})$ & $966 \pm 228$
\end{tabular}

${ }^{*} P<0.05$ compared to the vehicle-treated group. ${ }^{\ddagger} P<0.05$ compared with the corresponding (equimolar) dose of NCX 4215. $n=3-6$ per group. Tissues were incubated in vitro in the presence of the test drugs or vehicle. expectation was that the nitric oxide released from such a compound would protect the gastrointestinal tract from the damaging consequences of suppression of cyclo-oxygenase activity, as we have previously found with similar derivatives of flurbiprofen, ketoprofen and diclofenac (9-11). However, the aspirin derivative, NCX 4215, was found not to inhibit cyclo-oxygenase activity, but still to have significant inhibitory effects on platelet aggregation induced by thrombin (human platelets), collagen or ADP (rat platelets). In the human platelet studies, NCX 4215 was significantly more potent than aspirin as an inhibitor of aggregation. This augmented anti-aggregatory effect appeared to be related to release of nitric oxide from NCX 4215, since this compound significantly elevated platelet cGMP levels at concentrations that inhibited platelet aggregation, whereas aspirin did not, and because hemoglobin significantly attenuated the anti-aggregatory effects of NCX 4215 . Hemoglobin is a well characterized scavenger of nitric oxide (15) and has been shown in previous studies of this type to suppress nitric oxidemediated inhibition of platelet aggregation (13). Studies using chemiluminescent detection of nitric oxide confirmed that NCX 4215 released nitric oxide, but only when the drug was incubated with platelets (i.e., no spontaneous release). Nitric oxide release was observed within 1 min of addition of the compound to human platelets. These observations are consistent with the hypothesis that NCX 4215 must undergo metabolism by the platelet before releasing nitric oxide. In the case of platelet cGMP levels, statistically significant increases were not apparent with NCX 4215 after 1 min of incubation, but were observed with the 10-min incubation. Sodium nitroprusside, a well characterized nitric oxide donor, also increased platelet cGMP levels after $10 \mathrm{~min}$ incubation, but not after $1 \mathrm{~min}$.

In light of the observation that NCX 4215 does not inhibit cyclo-oxygenase activity (i.e., prostaglandin and thromboxane synthesis), it is perhaps not surprising that this compound did not produce significant damage in the stomach. There is abundant evidence supporting the hypothesis that suppression of gastric prostaglandin synthesis is a critical component of the ulcerogenic properties of aspirin and NSAIDs (20). Even after daily administration of this compound for $14 \mathrm{~d}$, gastric injury was not observed. Whether or not this compound will induce gastric injury when administered over a more prolonged period of time, or in other species, is the subject of ongoing studies.

Nitric oxide donors have been extensively characterized as inhibitors of platelet aggregation in vitro (12), but not in ex vivo or in vivo studies. One problem with the use of nitric oxide donors for anti-thrombotic applications is that drugs such as sodium nitroprusside and glyceryl trinitrate relax vascular smooth muscle, thereby reducing systemic blood pressure. Whether or not doses of nitric oxide donors below the threshold of effects on systemic blood pressure would release or generate sufficient nitric oxide to inhibit platelet aggregation has not been reported. It is noteworthy that at a dose that caused significant inhibition of collagen- and ADP-induced rat platelet aggregation ex vivo, intravenously administered NCX 4215 did not significantly affect systemic arterial blood pressure. On the other hand, an equimolar dose of sodium nitroprusside had no effect on platelet aggregation, but when given intravenously, caused profound hypotension and death within minutes. It is possible that the aspirin derivative releases nitric oxide at a slower rate and/ or in lower amounts that standard nitric oxide donors. Indeed, NCX 4215 caused a much smaller increase in platelet cGMP levels than that caused by an equimolar concentration of sodium 
nitroprusside. While our in vitro data suggest that NCX 4215 inhibits platelet aggregation at least in part through the release of nitric oxide, we cannot rule out the possibility that in vivo, the inhibition of platelet aggregation by this compound may occur through mechanisms unrelated to the generation of nitric oxide.

Aspirin is increasingly being used on a chronic basis as an anti-thrombotic agent. There is convincing evidence for beneficial effects of aspirin in the prevention of stroke and myocardial infarction $(1,6,22,23)$. Low doses of aspirin, when used over several days, are capable of profoundly suppressing platelet thromboxane synthesis and therefore platelet activation, while having only a modest inhibitory effect on gastric prostaglandin synthesis (24). Because of this, the incidence of gastrointestinal bleeding with low dose aspirin is much less than that observed with anti-inflammatory doses of aspirin or other NSAIDs. However, there remains a significant risk of gastrointestinal bleeding and hemorrhagic stroke with low dose aspirin $(23,25,26)$, as well as adverse effects related to blood pressure control in patients with essential hypertension $(27,28)$ and heart failure (29). Therefore, as recently concluded by Patrono (1), despite the effectiveness of aspirin in preventing between 20 and 33\% of "all important cardiovascular events," there remains a need for effective antithrombotic drugs which carry a lower risk of adverse effects. Since NCX 4215 is capable of inhibiting platelet aggregation at least as effectively as aspirin without affecting cyclo-oxygenase activity, it does not cause damage to the gastric mucosa. Since this compound does not suppress thromboxane synthesis, even when given daily for five days, it can be concluded that it is not metabolized to yield aspirin. It should be noted that even a conversion of $\sim 5 \%$ of the administered NCX 4215 to aspirin would have been sufficient to be detected through its ability to suppress thromboxane synthesis. The measurement of plasma salicylate levels using an assay that did not detect aspirin or NCX 4215 revealed that the majority ( 73\%) of the NCX 4215 administered was not converted to salicylate. Taken together with the observation that NCX 4215 was much more active than salicylate in terms of inhibiting platelet aggregation, we can rule out the possibility that the compound acted purely as a pro-drug for salicylate. While further studies are required to fully understand the metabolism and pharmacokinetics of this compound, NCX 4215 serves as a useful prototype to test the hypothesis that a nitric oxide donor can exert antithrombotic effects without untoward effects on systemic blood pressure or on the gastric mucosa.

\section{Acknowledgments}

Dr. Wallace is a Medical Research Council of Canada Scientist and an Alberta Heritage Foundation for Medical Research Scientist.

This work was supported by a grant from the MRC of Canada.

\section{References}

1. Patrono, C. 1994. Aspirin as an antiplatelet drug. N. Engl. J. Med. 330:1287-1294.

2. Fitzgerald, G. A. 1991. Mechanisms of platelet activation: thromboxane $A_{2}$ as an amplifying signal for other agonists. Am. J. Cardiol. 68:11B-15B.
3. Ware, J. A and D. D. Heistad. 1993. Platelet-endothelium interactions. $N$. Engl. J. Med. 328:628-635.

4. Radomski, M. W., R. M. J. Palmer, and S. Moncada. 1987. The antiaggregating properties of vascular endothelium: interactions between prostacyclin and nitric oxide. Br. J. Pharmacol. 92:639-646.

5. Kurata, J. H., and D. E. Abbey. 1990. The effect of chronic aspirin use on duodenal and gastric ulcer hospitalization. J. Clin. Gastroenterol. 12:260-266.

6. Steering Committee of the Physicians' Health Study Research Group. 1989. Final report on the aspirin component of the ongoing Physicians' Health Study. N. Engl. J. Med. 321:129-135.

7. Leivonen, M., P. Sipponen, and E. Kivilaakso. 1992. Gastric changes in coronary-operated patients with low-dose aspirin. Scand. J. Gastroenterol. 27:912-916.

8. Vane, J. R. 1971. Inhibition of prostaglandin synthesis as a mechanism of action for aspirin-like drugs. Nat. New Biol. 231:232-235.

9. Wallace, J. L., B. Reuter, C. Cicala, W. McKnight, M. Grisham, and G. Cirino. 1994. Novel nonsteroidal anti-inflammatory drug derivatives with markedly reduced ulcerogenic properties in the rat. Gastroenterology. 107:173-179.

10. Wallace, J. L., B. Reuter, C. Cicala, W. McKnight, M. Grisham, and G. Cirino. 1994. A diclofenac derivative without ulcerogenic properties. Eur. J. Pharmacol. 257:249-255.

11. Reuter, B. K., G. Cirino, and J. L. Wallace. 1994. Markedly reduced intestinal toxicity of a diclofenac derivative. Life Sci. 55:PL1-PL8.

12. Radomski, M. W., R. M. J. Palmer, and S. Moncada. 1987. Comparative pharmacology of endothelium-derived relaxing factor, nitric oxide and prostacyclin in platelets. Br. J. Pharmacol. 92:181-187.

13. Radomski, M., and S. Moncada. 1983. An improved method for washing human platelets with prostacyclin. Thromb. Res. 30:383-386.

14. Hogaboam, C. M., A. D. Befus, and J. L. Wallace. 1993. Modulation of rat mast cell reactivity by IL- $1 \beta$. Divergent effects on nitric oxide and plateletactivating factor release. J. Immunol. 151:3767-3774.

15. Martin, W., G. M. Villani, D. Jothianandan, and R. F. Furchgott. 1985 Selective blockade of endothelium-derived and glyceryl trinitrate-induced relaxation by hemoglobin and methylene blue in the rabbit aorta. J. Pharmacol. Exp. Ther. 232:708-716.

16. Palmer, R. M. J., A. G. Ferridge, and S. Moncada. 1987. Nitric oxide release accounts for the biological activity of endothelium-derived relaxing factor Nature (Lond.). 327:524-526.

17. Patrono, C., G. Ciabattoni, E. Pinca, F. Pugliese, G. Catrucci, A. De Salvo, M. A. Satta, and B. A. Peskar. 1980. Low dose aspirin and inhibition of thromboxane $\mathrm{B}_{2}$ production in healthy subjects. Thromb. Res. 17:317-327.

18. Wallace, J. L., and B. J. R. Whittle. 1985. Role of prostanoids in the protective actions of BW755C on the gastric mucosa. Eur. J. Pharmacol. 115:4552 .

19. Trinder, P. 1954. Rapid determination of salicylate in biological fluids Biochem. J. 57:310.

20. Wallace, J. L. 1993. Gastric ulceration: Critical events at the neutrophilendothelium interface. Can. J. Physiol. Pharmacol. 71:98-102.

21. Segasothy, M., S. A. Samad, A. Zulfigar, and W. M. Bennett. 1994 Chronic renal disease and papillary necrosis associated with the long-term use of nonsteroidal anti-inflammatory drugs as the sole or predominant analgesic. Am. J. Kidney Dis. 24:17-24.

22. The RISC Group. 1990. Risk of myocardial infarction and death during treatment with low dose aspirin and intravenous heparin in men with unstable coronary artery disease. Lancet. 336:827-830.

23. The SALT Collaborative Group. 1991. Swedish Aspirin Low-Dose Trial (SALT) of $75 \mathrm{mg}$ aspirin as secondary prophylaxis after cerebrovascular ischaemic events. Lancet. 338:1345-1349.

24. Lee, M., B. Cryer, and M. Feldman. 1994. Dose effects of aspirin on gastric prostaglandins and stomach mucosal injury. Ann. Intern. Med. 120:184189.

25. Meade, T. W., P. J. Roderick, P. J. Brennan, H. C. Wilkes, and C. C Kelleher. 1992. Extracranial bleeding and other symptoms due to low dose aspirin and low intensity oral anticoagulation. Thromb. Haemost. 68:1-6.

26. Cryer, B., G. Luk, and M. Feldman. 1995. Effects of very low doses of aspirin (ASA) on gastric, duodenal \& rectal prostaglandins (PGs) \& mucosal injury. Gastroenterology. 108:A77. (Abstr.)

27. Oates, J. A., G. A. Fitzgerald, R. A. Branch, E. K. Jackson, H. R. Knapp, and L. J. Roberts II. 1988. Clinical implications of prostaglandin and thromboxane $\mathrm{A}_{2}$ formation. N. Engl. J. Med. 319:761-767.

28. Patrono, C., and M. J. Dunn. 1987. The clinical significance of inhibition of renal prostaglandin synthesis. Kidney Int. 32:1-12.

29. Hall, D., H. Zeitler, and W. Rudolph. 1992. Counteraction of the vasodilator effects of enalapril by aspirin in severe heart failure. J. Am. Coll. Cardiol. 20:1549-1555. 